\title{
ESTUDIO DE LAS HERRAMIENTAS DE TRABAJO LÍTICAS DE UNA CABAÑA DE VALENCINA DE LA CONCEPCIÓN (SEVILLA)
}

\section{STUDY OF LITHIC TOOLS RECOVERED IN A PREHISTORIC HUT FROM VALENCINA DE LA CONCEPCIÓN (SEVILLA)}

\author{
por \\ OLGA SÁNCHEZ LIRANZO y \\ ALEJANDRO VERA FERNÁNDEZ
}

\section{INTRODUCCIÓN}

La industria lítica aquí analizada procede de la excavación de urgencia de la calle Alamillo, n³3 (Valencina de la Concepción), efectuada entre los meses de diciembre de 1997 y febrero de 1998. Los productos líticos pertenecen a una cabaña adscrita a lo que normativamente se ha denominado "Bronce Inicial"' (inicios del II ${ }^{\circ}$ milenio a. n. e.). Si aceptamos la división espacio-funcional del complejo arqueológico de Valencina de la Concepción-Castilleja de Guzmán, que comprende un espacio dedicado al poblado propiamente dicho, otro a la necrópolis y un tercero que se situaría en una zona intermedia, compuesta por silos, zanjas, etc., la estructura intervenida se encontraría entre el área intermedia y el área de poblado; pues al norte se sitúa el inmenso campo de silos de la Estacada Larga (Cruz-Auñón y Arteaga 1995) y al sur, integradas en el área intermedia, La Alcazaba (Cruz-Auñón y Arteaga 1997) y RTVA-El Cuervo (Arteaga y Cruz-Auñón 1995). La mencionada estructura se hallaba cortada por otra unidad estratigráfica, por lo que el material recuperado no pertenece a la totalidad de la cabaña.

Si finalmente aceptamos que la producción de instrumentos de trabajos sobre piedra mediante la talla es el resultado de una actividad humana para, obviamente, satisfacer determinadas necesidades, debemos intentar "inferir" qué necesidades cubrieron y de qué forma contribuyeron a la reproducción social.

Entendemos que el análisis de la industria lítica, por su particularidad, debe ser tratado desde una doble perspectiva:

- Como proceso de trabajo dedicado a la fabricación de herramientas líticas (producción).

- Como herramientas que, mediante su consumo, participan como instrumentos de producción en otros procesos de trabajo $^{2}$ (consumo).

1. Según la interpretación que el director de la excavación, Pedro Manuel López Aldana, desarrolló en el artículo recogido en el Anuario Arqueológico Andaluz de 1997 (en prensa), en el que nosotros/as ofrecimos el estudio preliminar de los productos líticos tallados.

2. Esta segunda perspectiva es determinante sobre la primera: la función de las herramientas de trabajo determinan su forma (Vargas 1990: 84). 
Antes de adentrarnos en este estudio quizás sea necesario tener en cuenta las siguientes cuestiones:

1) El conjunto lítico es muy reducido, impidiendo desarrollar análisis cuantitativos.

2) La gran mayoría de las piezas recuperadas están retocadas, lo que dificulta el análisis de las primeras fases de la producción de las herramientas de trabajo.

3) El conjunto lítico ofrecía un alto grado de fracturación ${ }^{3}$, alcanzando hasta un $80,95 \%$ del total.

4) No contamos con los medios técnicos y económicos necesarios para llevar a cabo análisis petrológicos y mineralógicos, así como estudios traceológicos a nivel microscópico, que nos habrían ayudado a definir las estrategias de abastecimiento de las materias primas y el consumo de los productos líticos.

\section{ESTUDIO DEL PROCESO DE FABRICACIÓN DE LAS HERRAMIENTAS DE TRABAJO LÍTICAS.}

En este apartado nos limitaremos a estudiar los recursos técnicos aplicados en la producción del conjunto lítico. Para ello seguiremos un esquema lógico, relacionado con las distintas fases implicadas en dicho proceso (ver fig. 1): selección de la materia prima (gestión de recursos); acondicionamiento de los núcleos; extracción de lascas y láminas; y por último, adecuación funcional de los productos líticos ${ }^{4}$. Este análisis, evidentemente, nos acercaría más a los procesos económicos y sociales, imbricados en el modo de producción de la sociedad en la que se produjo el conjunto.

\section{Tabla 1
INVENTARIO GENERAL DE LA INDUSTRIA LÍTICA TALLADA}

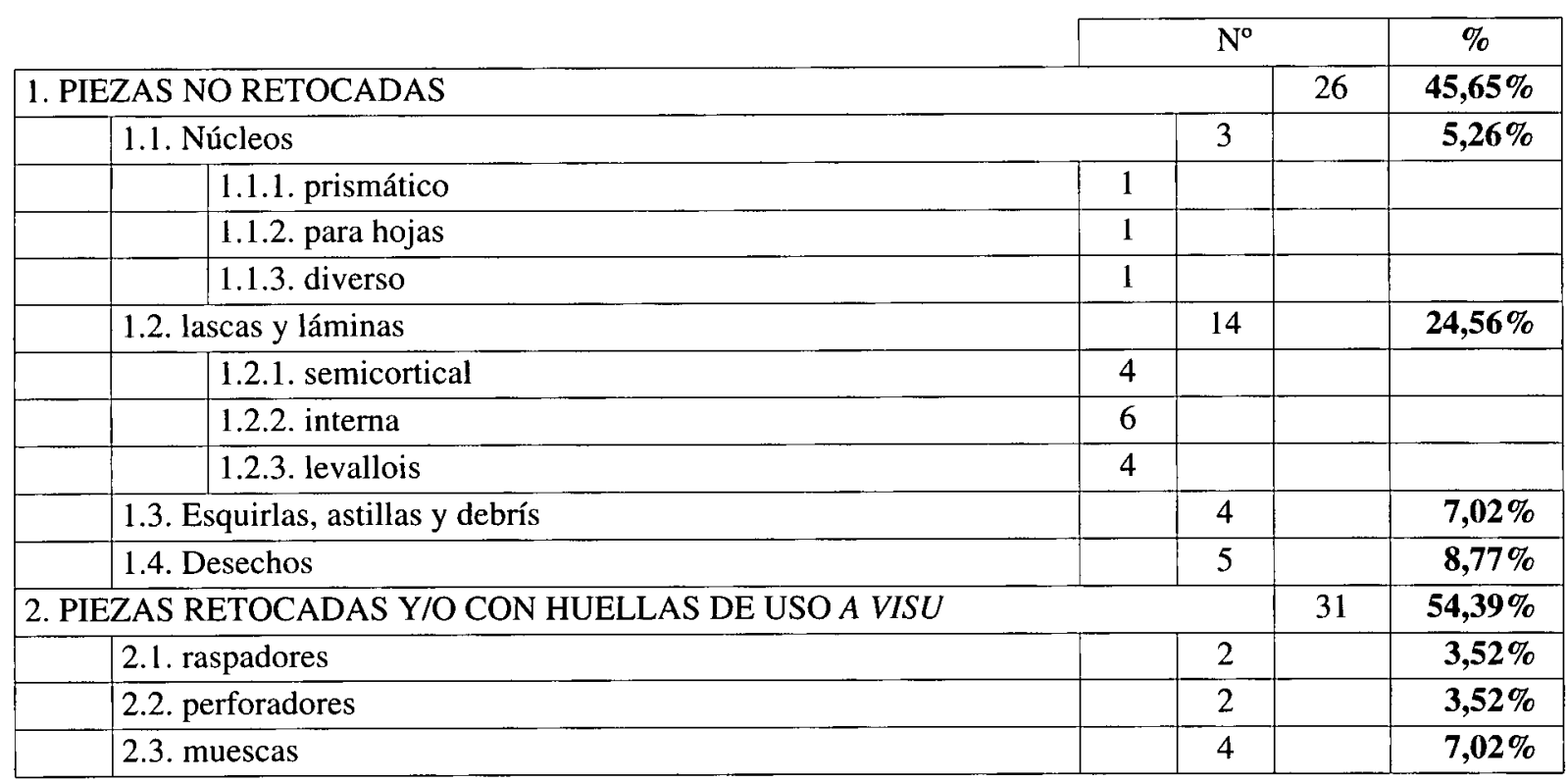

3. El alto grado de fracturación que presentan las piezas ha limitado nuestras posibilidades de análisis. La mayoría de las fracturas la hemos relacionado con los procesos de la talla, con procesos de consumo de los productos líticos o con procesos post-deposicionales, es decir consideramos son fracturas "no intencionales".

4. En este espacio pretendemos aproximarnos a la producción de instrumentos de trabajo de piedra a partir del análisis morfológico, técnico y tipológico. Somos conscientes de las limitaciones de este tipo de estudios, por tanto lo que presentamos no es más que un estudio "preliminar", un "esbozo", que tendrá que ser contrastado con el del resto del registro arqueológico hallado en la excavación y con otros análisis aplicados a la propia industria lítica. 


\begin{tabular}{|c|c|c|c|c|}
\hline & \multicolumn{3}{|c|}{$\mathrm{N}^{\circ}$} & $\%$ \\
\hline 2.4. denticulados & & 3 & & $5,26 \%$ \\
\hline 2.5. retoques simples & & 1 & & $\mathbf{3 , 2 3 \%}$ \\
\hline 2.6. retoques abruptos & & 4 & & $12,90 \%$ \\
\hline 2.7. Huellas de uso & & 9 & & $29,02 \%$ \\
\hline 2.10. retoques planos & & 3 & & $9,68 \%$ \\
\hline \begin{tabular}{l|l} 
2.10.1. puntas foliáceas \\
\end{tabular} & 3 & & & \\
\hline 2.11. varios & & 1 & & $3,23 \%$ \\
\hline \begin{tabular}{l|l|} 
& 2.11.1. retoques simples y abruptos \\
\end{tabular} & 1 & & & \\
\hline 2.12. Fractura retocada & & 1 & & $3,23 \%$ \\
\hline TOTAL & & & 57 & $100 \%$ \\
\hline
\end{tabular}

\subsection{SELECCIÓN DE MATERIAS PRIMAS}

Como apuntamos con anterioridad, no contamos con estudios petrológicos, ni mineralógicos, por ello, sólo podremos señalar a grandes rasgos las "preferencias" seguidas a la hora de seleccionar las materias primas que se usarán en la fabricación de las herramientas de piedra. Estas "preferencias" las analizaremos respecto a las piezas registradas: los núcleos, las lascas y láminas sin uso aparente y los "útiles", al no contar con información directa de los puntos de abastecimiento. Sobre esta cuestión únicamente tenemos constancia de la existencia de algunos guijarros o cantos pequeños de sílex en afloramientos del Aljarafe (Ramos 1991-92: 44). El uso de la cuarcita en el conjunto industrial se explicaría por la abundancia y calidad de esta materia en forma de cantos rodados de río en el entorno del valle del Guadalquivir.

Tabla 2

\begin{tabular}{|l|c|c|c|c|c|c|c|c|c|c|c|c|}
\hline \multicolumn{1}{|c|}{ M.P. } & \multicolumn{2}{|c|}{ Núcleos } & \multicolumn{2}{c|}{ Desechos } & \multicolumn{2}{c|}{ Esquirlas } & \multicolumn{2}{c|}{$\begin{array}{c}\text { Lascas-láminas } \\
\text { sin retocar }\end{array}$} & \multicolumn{2}{c|}{$\begin{array}{c}\text { Piezas } \\
\text { retocadas }\end{array}$} & \multicolumn{2}{c|}{ Total } \\
\hline & $\mathrm{N}^{\circ}$ & $\%$ & $\mathrm{~N}^{\circ}$ & $\%$ & $\mathrm{~N}^{\circ}$ & $\%$ & $\mathrm{~N}^{\circ}$ & $\%$ & $\mathrm{~N}^{\circ}$ & $\%$ & $\mathrm{~N}^{\circ}$ & $\%$ \\
\hline SÍLEX & 1 & $33,33 \%$ & 4 & $80 \%$ & 1 & $25 \%$ & 9 & $64,28 \%$ & 25 & $80,64 \%$ & 40 & $70,18 \%$ \\
\hline CUARCITA & & & 1 & $20 \%$ & & & 2 & $14,29 \%$ & 4 & $12,90 \%$ & 7 & $12,28 \%$ \\
\hline CALIZA & & & & & & & 1 & $7,14 \%$ & 1 & $3,23 \%$ & 2 & $3,51 \%$ \\
\hline CUARZO & 2 & $66,67 \%$ & & & 3 & $75 \%$ & 2 & $14,29 \%$ & 1 & $3,23 \%$ & 8 & $14,03 \%$ \\
\hline TOTAL & 3 & $100 \%$ & 5 & $100 \%$ & 4 & $100 \%$ & 14 & $100 \%$ & 31 & $100 \%$ & 57 & $100 \%$ \\
\hline
\end{tabular}

Las rocas silíceas son las materias primas más abundantes del conjunto, llegando a representar el $70.17 \%$ del total. Le siguen, aunque a gran distancia, el cuarzo y la cuarcita con un $14.04 \%$ y un $12.28 \%$ respectivamente. También hay que indicar la presencia de dos piezas sobre rocas calizas (fig. $2, \mathbf{n}^{\circ} 2$ ).

Los sílex son muy variados, desde unos con escasa calidad, hasta otros cuya calidad permite que la talla sea muy precisa. Los colores son también muy diversos, los más comunes son los grises y pardos en distintas tonalidades, además de los blancos, negros y rosas (algunos de ellos translúcidos).

La cuarcita se encuentra representada con un $12.28 \%$. Esta materia prima es de muy buena calidad y sus colores son grises, pardos y cremas fundamentalmente. Curiosamente el cuarzo tiene una mayor presencia que la cuarcita con un $14.04 \%$. Algunas de estas piezas sobre cuarzo están tan alteradas que 
difícilmente podremos determinar si su fractura es intencional o natural, e incluso en algunos casos, si ésta es reciente o antigua.

Muy significativo nos parece el predominio del sílex en las lascas-láminas $\sin$ retocar $^{5}(64.28 \%)$ y en las piezas retocadas ${ }^{6}(80.64 \%)$. Muy escaso es el número de núcleos, desechos y esquirlas. Por otra parte, de los tres núcleos encontrados sólo uno está elaborado sobre roca silícea, a pesar de que esta materia prima es dominante, sin embargo hemos de destacar que de los cinco desechos de núcleos, cuatro son de sílex.

Como ya adelantamos anteriormente, en cuarcita apenas tenemos un total de siete piezas, de las cuales dos son lascas-láminas sin retocar y cuatro retocadas (dos de ellas puntas foliáceas, lo que supone un dominio manifiesto de la talla sobre este tipo de material). Esta escasez es llamativa teniendo en cuenta que dicha materia prima es muy abundante en este territorio y muy accesible a los grupos humanos.

En cuarzo hay ocho piezas, dos lascas-láminas, una pieza retocada y 3 esquirlas. La presencia de la caliza es meramente testimonial (una pieza retocada).

El material presenta un rodamiento muy leve, sólo dos piezas tienen un rodamiento algo más serio.

\subsection{PROCESOS RELACIONADOS CON LA PREPARACIÓN DE LOS NÚCLEOS Y LA EXTRACCIÓN DE LAS BASES POSITIVAS.}

\subsubsection{Núcleos.}

Hemos de tener en cuenta que sólo contamos con tres núcleos (prismático, para hojas y diverso). Por esta razón, la mayoría de la información que tenemos de los núcleos la hemos obtenido de las "huellas" visibles que quedan en las lascas y láminas extraídas. Así que para abordar la estrategia seguida en el acondicionamiento de los núcleos, hemos considerado dos cuestiones:

1. La preparación de la plataforma de percusión (parte de la cual queda conservada en las lascas y láminas -talones-). Hay que aclarar que para el análisis de los talones, hemos incluido la totalidad de lascas y láminas (tanto retocadas como sin retocar). Es destacable el predominio de talones abatidos $(60 \%)$. Dentro de los talones reconocibles destacan los lisos, que suponen casi las tres cuartas partes $(72,22 \%)$. A los lisos le siguen los talones corticales y los puntiformes (fig. 2, $\mathrm{n}^{\circ} 3$ ).

2. Las características de las primeras piezas extraídas. Sólo el $24,14 \%$ de las piezas documentadas conservan córtex parcialmente. De ello se desprende: primero, que las primeras fases del procesado no se documentan en la estructura estudiada (o en sus aledaños); y segundo, que no se observa un aprovechamiento generalizado de las primeras Bases Positivas extraídas de los "nódulos".

\subsubsection{Tipometría general de la industria.}

No ha sido posible determinar la tendencia laminar de la industria, debido tanto a las numerosas piezas fracturadas, como al elevado porcentaje de piezas retocadas.

Sin embargo, hemos decidido aplicar el análisis dimensional propuesto por Bagolini (1968), sólo a modo de referencia o como dato orientativo de la tipometría de la industria. Únicamente se han podido adscribir 17 piezas incluyendo las retocadas ${ }^{7}$ :

5. Clasificadas según el Sistema Lógico Analítico (SLA) como Bases Positivas (Mora et alii 1992).

6. Clasificadas según el SLA como Bases Negativas de Segunda Generación (Mora et alii 1992).

7. Pese a utilizar el modelo de estudio tipométrico ofrecido por Bagolini (1968) aplicable solamente a los "restos de talla", hemos considerado la industria lítica de forma global como anteriormente harían José Ramos Muñoz (1991-92: 46) y Olga Sánchez Liranzo (1997). No ignoramos la limitación que supone este recurso, y el carácter no definitivo que adquieren los 
Tabla 3:

PIEZAS SIN RETOCAR

\begin{tabular}{|l|l|}
\hline MICROLASCA & 1 \\
\hline MICROLASCA LAMINAR & 2 \\
\hline MICROLASCA ANCHA & 1 \\
\hline LASCA & 1 \\
\hline TOTAL & 5 \\
\hline
\end{tabular}

Tabla 4:

PIEZAS RETOCADAS

\begin{tabular}{|l|c|}
\hline MICROLASCA ANCHA & 2 \\
\hline MICROLÁMINA & 1 \\
\hline LASCA & 2 \\
\hline PEQUEÑA LASCA LAMINAR & 1 \\
\hline PEQUEÑA LASCA & 3 \\
\hline GRAN LASCA & 2 \\
\hline GRAN LÁMINA ESTRECHA & 1 \\
\hline TOTAL & 12 \\
\hline
\end{tabular}

Como podemos observar, se trata de un conjunto lítico con una tendencia a los tamaños reducidos, a tenor de la escasa presencia de ejemplares "macrolíticos" (sólo 2 grandes lascas y 1 gran lámina estrecha). No ignoramos que de alguna forma el tamaño de piezas, puede estar determinado, entre otros aspectos, por el tipo de materia prima utilizada.

\subsubsection{Técnicas aplicadas en la talla.}

La talla mediante percusión (directa o indirecta) queda atestiguada por alguna pieza, que por sus dimensiones y por la materia prima utilizada hace pensar en la utilización de un percutor duro. Tampoco descartamos el uso de la talla mediante percutor blando para los retoques de algunas de las piezas.

La talla a presión podría quedar representada por un núcleo prismático y otro para hojas, por la presencia de 10 hojas y por la documentación de dos talones puntiformes que podrían sugerirnos una preparación especial de la plataforma de percusión, destinada a una talla controlada por presión.

resultados. Pero no encontramos otra posibilidad mejor, puesto que el escaso número de piezas cuantificadas no permitía la utilización de métodos estadísticos (Mora et alii 1992). 


\subsection{LASCAS Y LÁMINAS SIN RETOQUE.}

Tenemos un total de 23 piezas sin retocar, que según el Sistema Lógico Analítico se encuadraría en la definición de las Bases Positivas. Dentro de este conjunto se podrían establecer dos grupos:

a) Aquellas Bases Positivas, que no presentan sus filos retocados, pero que fueron con seguridad utilizadas como herramientas de trabajo. Aquí se incluirían las lascas y láminas que presentan huellas de uso a visu. Se han documentado un total de 9 piezas con huellas apreciables de su utilización ${ }^{8}$, de las cuales el $66.67 \%$ son lascas-láminas internas y el $33.33 \%$ son lascas-láminas semicorticales.

b) Aquellas lascas-láminas sin retocar que no presentan huellas de uso apreciables a visu, y que constituyen un total de 14 piezas. Entre las lascas y las láminas (estas últimas sólo representadas por dos ejemplares), predominan las internas $(42,86 \%)$, mientras que las semi-corticales y las levallois quedan representadas en un $28,57 \%$, en ambos casos (fig. $2, n^{\circ} 3$ ).

En función del contexto arqueológico en el que se documentaron estas piezas líticas, consideramos que tanto unas como otras fueron "consumidas" como herramientas de trabajo, aunque tan sólo nueve de ellas confirmen de forma evidente y clara su uso ${ }^{9}$.

\subsection{ADECUACIÓN FUNCIONAL (Y TIPOLÓGICA ${ }^{10}$ ) DE LOS SOPORTES.}

Las distintas funciones o trabajos que realizan estas herramientas de piedra van a necesitar una distinta morfología, lo que les permite adecuarse a las tareas en las que se insertan como parte de los medios de producción. Unas morfologías que en muchos casos se "estandarizan" como nos demuestran las tipologías líticas establecidas.

Desde nuestra perspectiva, el problema no está en admitir que hay una relación entre determinados "tipos" que se repiten y unas "actividades" concretas; sino en descubrir cuál es la naturaleza de esa relación. Lógicamente esta cuestión ha de recaer fundamentalmente en los estudios de las huellas de uso (microscópicas y macroscópicas) que quedan en las piezas y su asociación con el resto del registro arqueológico. Por todo esto, nos parece importante indicar que las "tipologías" tienen un papel importante en los estudios de las industrias líticas, aunque también abogamos por la necesaria revisión de los análisis tipológicos que se vienen utilizando en el estudio de estos materiales arqueológicos. Es necesario establecer una relación dialéctica entre los aspectos funcionales, tipológicos y morfológicos de la industria lítica.

Si agrupamos las piezas que estamos analizando en unos "tipos" concretos que se repiten y que responden a una evidente tradición técnica e histórica, salen a colación algunas cuestiones interesantes. Una de ellas es que casi la mitad de las piezas que tradicionalmente se denominan "útiles" (piezas retocadas), no se amoldan a unas morfologías y transformaciones concretas que nos permitan clasificarlas dentro de las listas tipológicas. Son piezas que apenas han sido transformadas y que sólo sus filos se han modificado mediante retoques ". Dentro de este grupo hay seis piezas con retoques simples y abruptos cuyo soporte

8. Generalmente se han denominado a estas huellas "retoques de uso" (un retoque muy marginal y continuo). Este tipo de huellas no es accidental, ni intencional, sino que es el producto del contacto del filo de la pieza con la materia trabajada. Por tanto, no consideramos adecuado definir estas huellas como "retoque", porque no son el resultado del proceso de fabricación del útil, sino de su consumo.

9. Aunque estas piezas han sido tradicionalmente clasificadas como "restos de talla", no hemos de olvidar que muchas de ellas fueron utilizadas directamente, sin retoque previo, aunque no se aprecien a simple vista las huellas de su consumo.

10. En este trabajo, seguiremos la clasificación tipológica propuesta por Laplace $(1975 ; 1985)$

11. Hemos decidido incluir en este apartado sobre la tecnología, los tipos de retoque, puesto que el retoque debe considerarse una característica tecnológica. 
es siempre una lámina interna (hoja), permitiendo obtener unos filos homogéneos. Los retoques están situados en los filos laterales de las piezas (izquierda y/o derecha), sólo una tiene retocada también el extremo distal y proximal.

El $85,71 \%$ de las piezas retocadas (22) se han realizado sobre soportes internos. Queda atestiguado un predominio del retoque abrupto (fig. $4, \mathrm{n}^{\circ} 4$ y 5), tradicionalmente vinculado a la preparación de las piezas para su posterior enmangue o su más fácil sujeción; seguido por el retoque plano, utilizado para las piezas foliáceas (tan características de esta época); mientras que el retoque simple muestra una presencia meramente testimonial (ver tabla 1 ).

Un grupo importante lo forma el de las muescas (fig. $4, \mathrm{n}^{\circ} 2$ ) y los denticulados (fig. $4, \mathrm{n}^{\circ} 1$ ) que suman siete piezas $(31.81 \%)$. Las cuatro muescas tienen retoques abruptos y simples, marginales, continuos y directos y/o inversos. Tres presentan huellas de uso en alguno de sus filos. Todas las muescas han usado la roca silícea como materia prima, una sobre lámina interna, dos sobre lasca interna y una sobre desecho de núcleo. Esta variabilidad en el soporte indica que no existe una estandarización en la fabricación de este tipo de herramientas, al menos en cuanto al tipo de soporte empleado.

Piezas denticuladas tenemos tres: dos en sílex y una en cuarcita. Los retoques predominantes son abruptos, marginales y directos, situados todos ellos en los filos derechos e izquierdos. Aquí también encontramos cierta variabilidad en los soportes usados: lascas semicorticales, lascas internas y lasca-núcleo (fig. 3).

Otro conjunto de piezas interesantes, por su larga tradición técnica e histórica son aquellas que se clasifican dentro de los raspadores y los perforadores. Aquí contamos con dos raspadores, uno de ellos es un raspador simple (G-11) que está fabricado sobre lasca interna, presentando huellas de uso en el lateral izquierdo. El otro usa como soporte un desecho de núcleo cuyo grosor nos permite clasificarlo en el grupo de los carenados y en el de los raspadores en hocico (G-22) (fig. 3, $\mathrm{n}^{\circ} 7$ ).

Contamos con dos perforadores, uno de ellos está realizado sobre una lámina interna (fig. $3, n^{\circ} 8$ ), consiguiendo su configuración mediante retoques planos y abruptos, profundos, directos y continuos en el filo izquierdo y proximal. El otro perforador usa como soporte un desecho que aún tiene algún resto de córtex, el retoque que se sitúa en el lateral izquierdo y derecho y se podría describir como simple, marginal, continuo y denticulado, también encontramos sobre él huellas de uso.

También contamos con una fractura retocada sobre lasca interna con retoques abruptos, marginales, directos y continuos.

Como tipo relativamente reciente (a partir del $\mathrm{III}^{\circ}$ milenio a.n.e.) tenemos un elemento de hoz que está fabricado sobre lámina interna (fig. $5, \mathrm{n}^{\circ}$ 5). Éste presenta un borde denticulado bifacial recto. Siguiendo la ficha propuesta por el profesor Dr. José Ramos Muñoz se clasificaría en el tipo 1.19 (1990-91).

También contamos con tres puntas de flecha foliáceas. Dos de ellas están elaboradas sobre cuarcita y se clasificarían-siguiendo el esquema tipológico propuesto por Bagolini-como F2A (puntas foliáceas con alerones), ambas poseen la base cóncava y tienen fracturada la punta (fig. $5, \mathrm{n}^{\circ} 3$ y 4 ). La tercera (fig. $5, \mathrm{n}^{\circ} 2$ ), fabricada en sílex, no posee alerones y se clasificaría, siguiendo el mismo esquema, como F2B (punta foliácea con base simple) (Merino 1994: 193-196).

\section{APROXIMACIÓN AL CONSUMO (USO) DE LAS HERRAMIENTAS DE TRABAJO DE PIEDRA.}

Los productos líticos tallados se hacen "productos" cuando son usados o consumidos por los seres humanos, es decir cuando se insertan en otros procesos de trabajo.

Como repetidamente venimos exponiendo, no contamos con estudios funcionales microscópicos, así que la asociación o clasificación que hemos realizado entre unos "tipos" y unos "trabajos" debe ser tomada 
de forma "provisional", hasta que análisis más rigurosos y profundos nos permitan determinar claramente los trabajos que estas herramientas realizaban, así como los materiales sobre los que se usaban. Nosotros/as hemos agrupado las herramientas en función de los procesos de trabajos en los cuales consideramos que estas herramientas se integran ${ }^{12}$ :

\section{- Herramientas de trabajo relacionadas con las actividades agrícolas.}

En este grupo incluimos el elemento de hoz encontrado y que suele usarse en la recogida de los cereales, es decir en la siega. Este tipo de piezas formarían parte de un útil compuesto (hoz) constituido por un mango de un material de difícil conservación (madera o hueso) en cuyo filo se insertarían mediante una ranura en el mango estas piezas (elementos de hoz) que se fijarían seguramente con resina.

En relación con los tres denticulados encontrados hay que decir que también se han asociado con el trabajo de la siega, de hecho se plantea que estos van a ser sustituidos más adelante por los elementos de hoz como ha indicado alguna investigadora (Valverde 1993).

Hay también quien relaciona el trabajo de la siega con las lascas y láminas que presentan huellas de uso (Vaugham y Bouquet 1987: 504) que en este conjunto suman un total de nueve piezas. En relación con esto, José Ramos sugirió que las dimensiones y la alta fracturación de un conjunto lítico procedente de Valencina de la Concepción, podrían indicar que dichas piezas fueron usadas a modo de "hoces" (Ramos 1991-92: 57).

\section{- Herramientas de trabajo relacionadas con las actividades de caza.}

Las tres puntas de flechas foliáceas son las herramientas que presentan una funcionalidad más clara y evidente. Serían piezas apuntadas mediante retoques planos que debieron estar engarzadas a una vara de madera. A pesar de todo también hay quiénes plantean que estas puntas pudieron servir como "puntas de dardos arrojadizos". Algunos estudios traceológicos de las puntas han demostrado que algunas de ellas fueron reutilizadas para otras funciones para las que fueron fabricadas inicialmente como raspar el hueso y el asta (Geneste y Plisson 1990: 304).

\section{- Herramientas de trabajo relacionadas con las actividades artesanales (de la madera, pieles, hueso,...).}

En este grupo de herramientas incluimos los dos raspadores. De forma general los estudios funcionales realizados por distintos investigadores relacionan este tipo de útiles con la preparación de las pieles (Plisson 1985; Mansur-Franchomme 1986). Otros, sin embargo, señalan que esto no debe ser tomado como una regla porque de hecho algunos raspadores se han asociado con el trabajo de la madera y del hueso, aunque siempre usado de forma transversal (Clemente y Terradas 1993: 519).

Dentro de este conjunto incluimos dos perforadores cuya funcionalidad es evidente: la realización de orificios mediante movimientos giratorios. Mucho más complejo resulta averiguar sobre qué materiales han sido empleados (hueso, madera, asta, cerámica, ...).

Las dos muescas retocadas podrían haberse empleado para raer o apuntar materiales como la madera, el hueso o el asta (Tixier 1976), aunque hay quien lo cuestiona (Vaughan y Bouquet 1987: 403).

12. Para realizar esta aproximación "provisional" de los usos o consumos de las herramientas de trabajo nos hemos apoyado en la tesis de licenciatura de Manuela Pérez Rodríguez (1997). 


\section{- Herramientas de trabajo relacionadas con el procesado de los alimentos.}

Las seis piezas que se han clasificado como piezas con retoques simples y/o abruptos son más difíciles de encuadrar funcionalmente. En algunos casos, los retoques abruptos de las piezas nos indicaría no la zona funcional, sino la del "enmangue", siendo su filo opuesto el usado aunque no presente retoques. Estas herramientas se pudieron usar como cuchillos para el procesado de los alimentos, las actividades de carnicería, etc.

\section{CONCLUSIONES:}

- Aunque la cabaña se ha adscrito al primer tercio del $\mathrm{II}^{\circ}$ Milenio a.n.e. -siguiendo principalmente la lectura crono-cultural ofrecida por la industria cerámica-, no hemos de olvidar que el conjunto lítico aquí ofrecido no es exclusivo ni definitorio de este período, sino que abarcaría todo el $\mathrm{III}^{\mathrm{er}}$ milenio y buena parte del II $^{\circ}$ milenio a.n.e.

- Es probable que la mayoría de las herramientas líticas talladas se hayan fabricado sobre materias primas del entorno. Sin embargo, no descartamos que algunas piezas silíceas muy concretas puedan proceder de un centro de abastecimiento exógeno.

- La talla de la industria se realizaría en un lugar ajeno a la estructura analizada, puesto que no contamos con ninguna pieza de descortezado, la presencia de los núcleos es meramente testimonial y son muy escasos los desechos, astillas, debrís y esquirlas.

- Nos encontramos en un contexto de consumo de productos líticos tallados. De hecho, más de la mitad de las piezas documentadas están retocadas o poseen huellas de su uso a visu. Además, a éstas debemos sumar aquellas lascas y láminas que, aunque no presentan retoques ni huellas apreciables a simple vista de su utilización, pudieron haber sido consumidas como herramientas de trabajo. Futuros estudios traceológicos podrían ayudarnos a conocer el tipo de actividad que desarrollaron estas herramientas (algunas de las cuales fueron propuestas en el apartado $3^{\circ}$ de este artículo a modo de hipótesis). Sólo así, podremos aproximarnos a los modos de producir y de vivir de las formaciones sociales y económicas que habitaron el entorno aljarafeño durante los primeros siglos del $\mathrm{II}^{\circ}$ milenio a.n.e.

- En este conjunto se evidencia claramente un dominio general (conocimiento y experiencia) de las distintas técnicas de talla que se requieren para la producción de herramientas de trabajo, desde las más simples a las más complejas. Por ello, no descartamos que el proceso de elaboración de estas piezas pudiese estar en manos de ciertos/as artesanos/as especializados/as. 


\section{BIBLIOGRAFÍA}

ARTEAGA, O. y CRUZ-AUÑÓN, R. (1995): “Una valoración del "Patrimonio Histórico" en el "Campo de Silos" de la finca "El Cuervo- RTVA" (Valencina de la Concepción, Sevilla). Excavación de urgencia de 1995". Anuario Arqueológico de Andalucía, 1995: 608-616. Consejería de Cultura, Junta de Andalucía, Sevilla.

BAGOLINI, B. (1968): "Ricerche sulle dimensioni dei manufatti litici prehistorici non ritocatti", Annali dell'Université di Ferrara. Sezione XV, vol. I, n¹0: 195-219. Ferrara.

CLEMENTE, I. y TERRADAS, X. (1993): “Matiéres premieres et fonctions: l'exemple de l'outillage lithique des Yamanas (Terra de Feu)", Traces et fonction: les gestes retrouvés, Colloque Internacional de Liège, Eraul, vol. 50.

CRUZ-AUÑÓN, R. y ARTEAGA, O. (1995): “Acerca de un campo de silos y un foso de cierre prehistóricos ubicados en "La Estacada Larga" (Valencina de la Concepción, Sevilla). Excavación de urgencia de 1995". Anuario Arqueológico de Andalucía, 1995: 600-607. Consejería de Cultura, Junta de Andalucía, Sevilla.

CRUZ-AUÑÓN, R. y ARTEAGA, O. (e.p.): "Intervención arqueológica de urgencia en la Urbanización "La Alcazaba" (Valencina de la Concepción, Sevilla). Campaña 1997", Anuario Arqueológico de Andalucía, 1997. Consejería de Cultura, Junta de Andalucía, Sevilla.

GENESTE, J.M. y PLISSON, H. (1990): "Technologie fonctionelle des pointes a cran solutreennes: l' apport des nouvelless donnes de la grotte de Combe Sauniere (Dordogne)", Eraul 42. Liège.

LAPLACE, G. (1975): “La Typologie analythique et structurale: Base rationnelle d'ètude des industries lithiques et osseuses” Banque de Données Archeologiques. Marsella juin 1972. C.N.R.S. Paris.

LAPLACE, G. (1986): Tipología Analítica. Facultad de Filología y de Geografía e Historia. Departamento de Prehistoria y Arqueología de la Universidad de Vitoria.

LÓPEZ ALDANA, P. et alii (en prensa): "Excavación de urgencia de una estructura habitacional en Valencina de la Concepción (Sevilla)", Anuario Arqueológico Andaluz 1997. Sevilla.

MANSUR-FRANCHOMME, M.E. (1986): Microscopie du materiel lithique préhistorique. C.N.R.S. Paris.

MERINO, J. Ma. (1994): “Tipología lítica”. $3^{a}$ Edición. Munibe, suplemento no 9. San Sebastián. Sociedad de Ciencias Aranzadi.

MORA, R.; MARTÍNEZ, J. y TERRADAS, X. (1992): “Un proyecto de análisis: el Sistema Lógico Analítico (S.L.A.)", en R. Mora et alii (ed.): Tecnología y Cadenas Operativas Líticas, Treballs d'Arqueologia: 173-200. Barcelona.

PÉREZ RODRÍGUEZ, M. (1997): La producción de instrumentos líticos pulimentados en la prehistoria reciente de la Banda Atlántica de Cádiz. Servicio de Publicaciones de la Universidad de Cádiz.

PLISSON, H. (1985): "Contribution de la tracéologie a la localisation des aires d'activité et d'occupation", L'Anthropologie, t. 89, nº 4: 473-478. Paris.

RAMOS, J. (1991-92): "La industria lítica del Cobre Pleno del poblado de Valencina de la Concepción (Sevilla)", Norba, 11-12: 43-74.

SÁNCHEZ LIRANZO, O. (1997): “Estudio de "productos" líticos tallados de la calle Dolores Quintanilla (Carmona, Sevilla)", Spal 6: 35-51. Sevilla.

TIXIER, J. (1976): "L'industrie lithique capsienne de l'Aïn Dokkara, región de Tebessa, Algérie", Libyca XXIV: 21-53. Alger.

VALVERDE, M. (1993): El taller de Cantarranas (El Puerto de Santa María, Cádiz). Un ejemplo para la transición Neolítico/Calcolítico. Servicio de Publicaciones. Universidad de Cádiz.

VARGAS, I. (1990): Arqueología, ciencia y sociedad. Ensayo sobre teoría arqueológica y la formación económico social tribal en Venezuela. Abre Brecha. Caracas.

VAUGHAN Y BOUQUET, A. (1987): "Première étude fonctionnelle d'outils lithiques néolithiques du village de Charaviens, Isère", L'Anthropologie, t. 91, n² 2: 399-410. Paris. 


\section{Producción y consumo de la industria lítica}

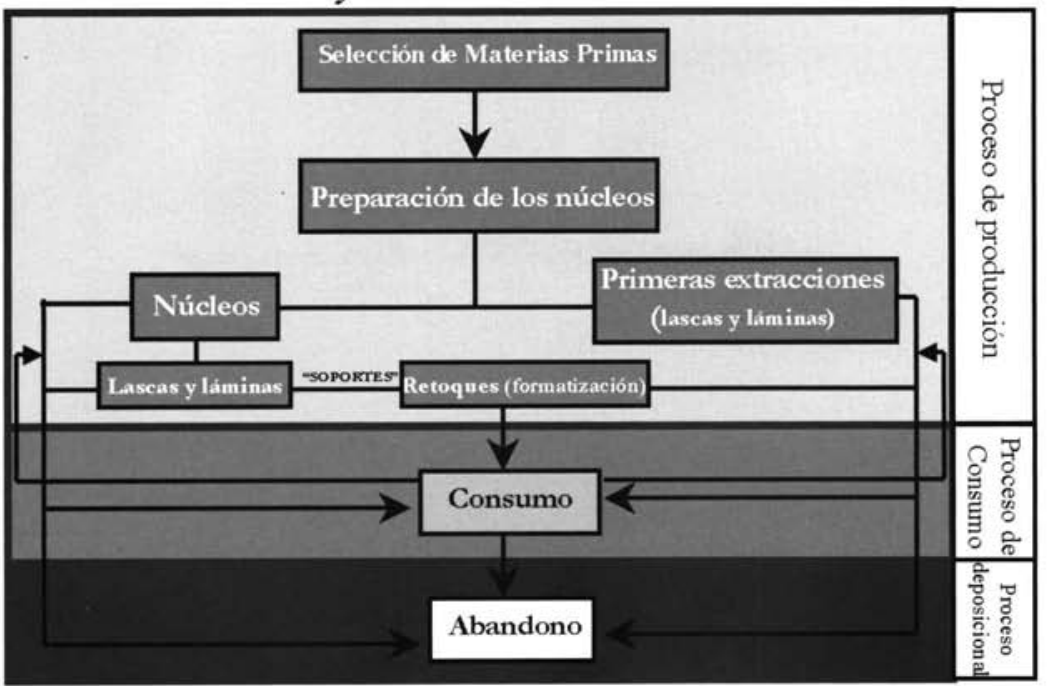

FIGURA 1:

1. Cuadro 1: Los procesos de producción y consumo de los productos líticos tallados. 


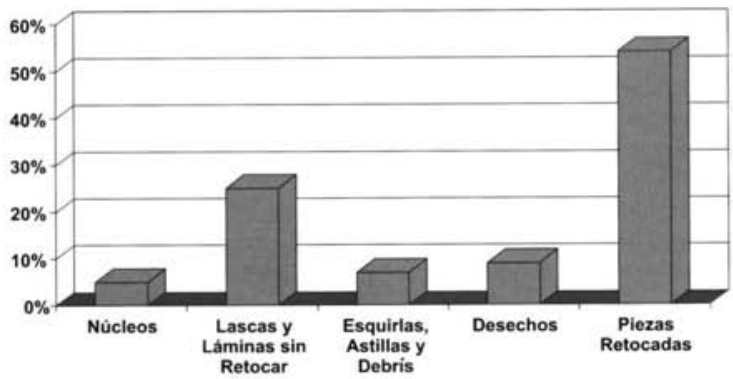

1. Gráfica 1: Cómputo General de la industria lítica

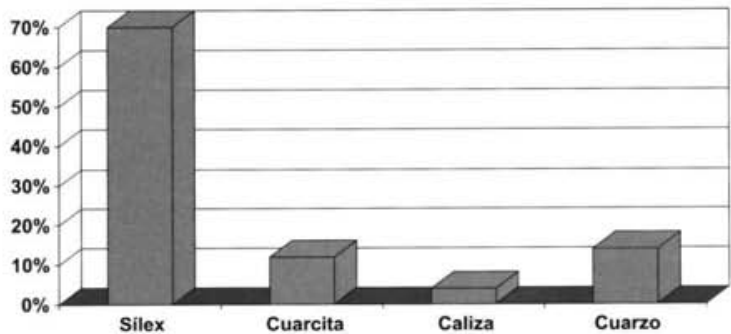

2. Gráfica 2: Materias primas utilizadas.

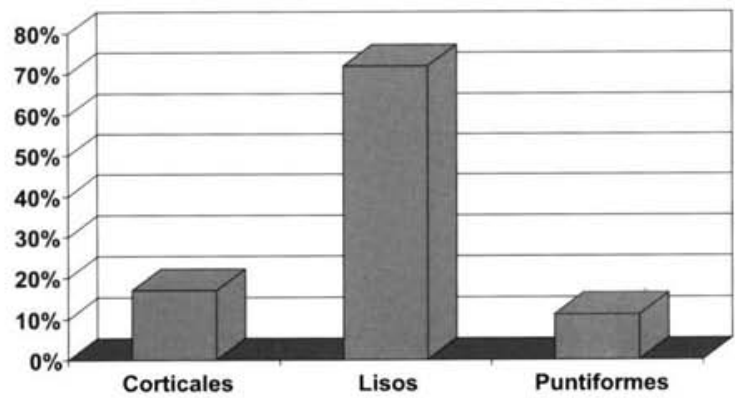

3. Gráfica 3: Tipos de talones reconocibles (no se especifican los abatidos).

FIGURA 2: 

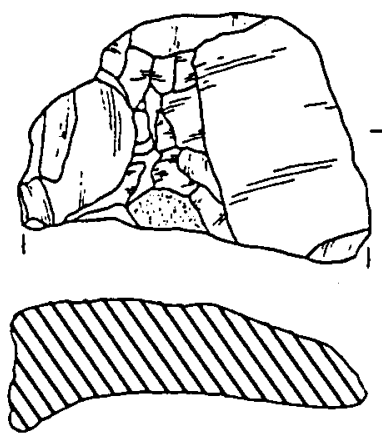

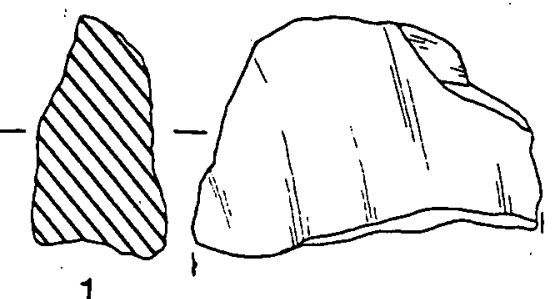

1
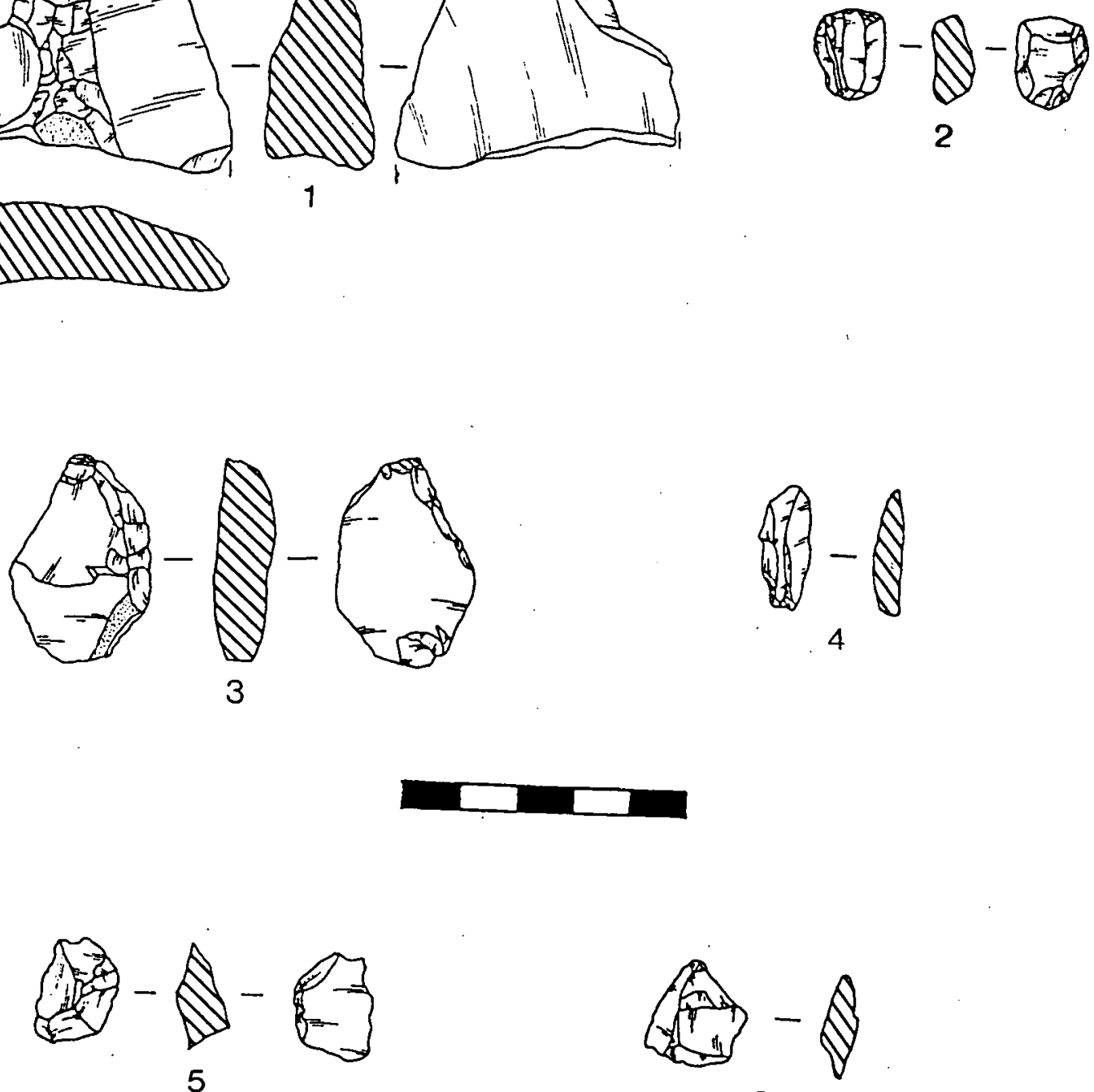

in

6
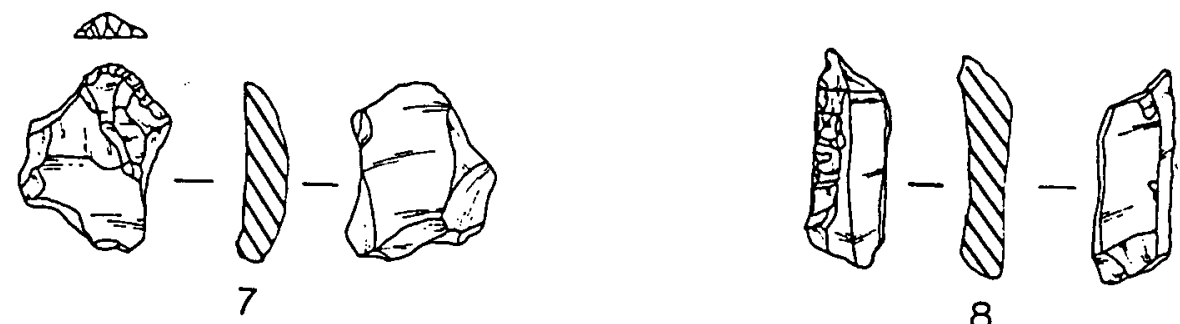

FIGURA 3: 1.- Desecho de núcleo. 2.- Núcleo para laminitas. 3.- Lasca semicortical. 4.- Lámina interna. 5 y 6.- Lascas levallois. 7.- Raspador en hocico (G22). 8.- Perforador. 

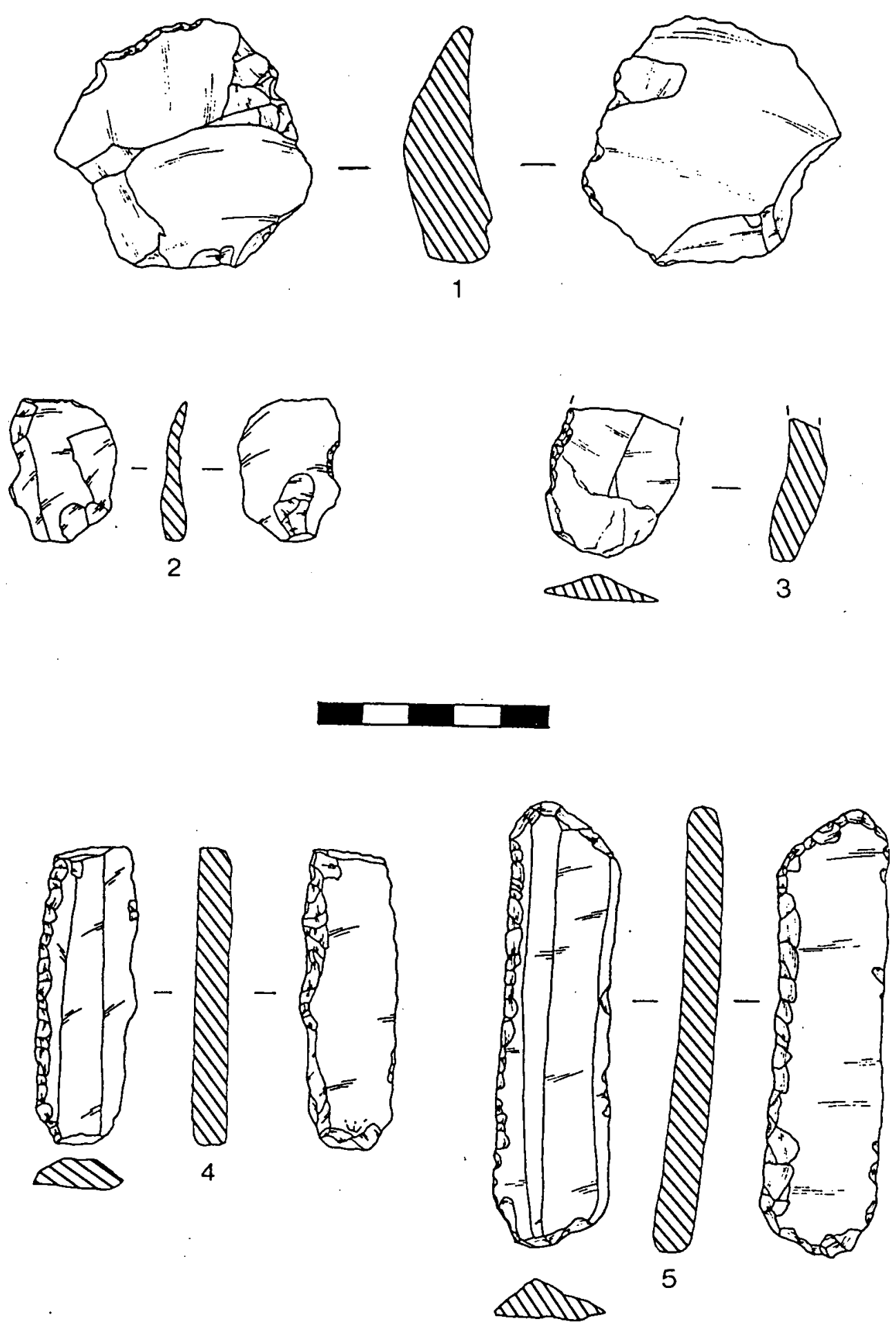

FIGURA 4: 1.- Lasca denticulada. 2.- Muesca retocada. 3.- Lasca con retoque simple. 4 y 5.- Láminas con retoque abrupto. 

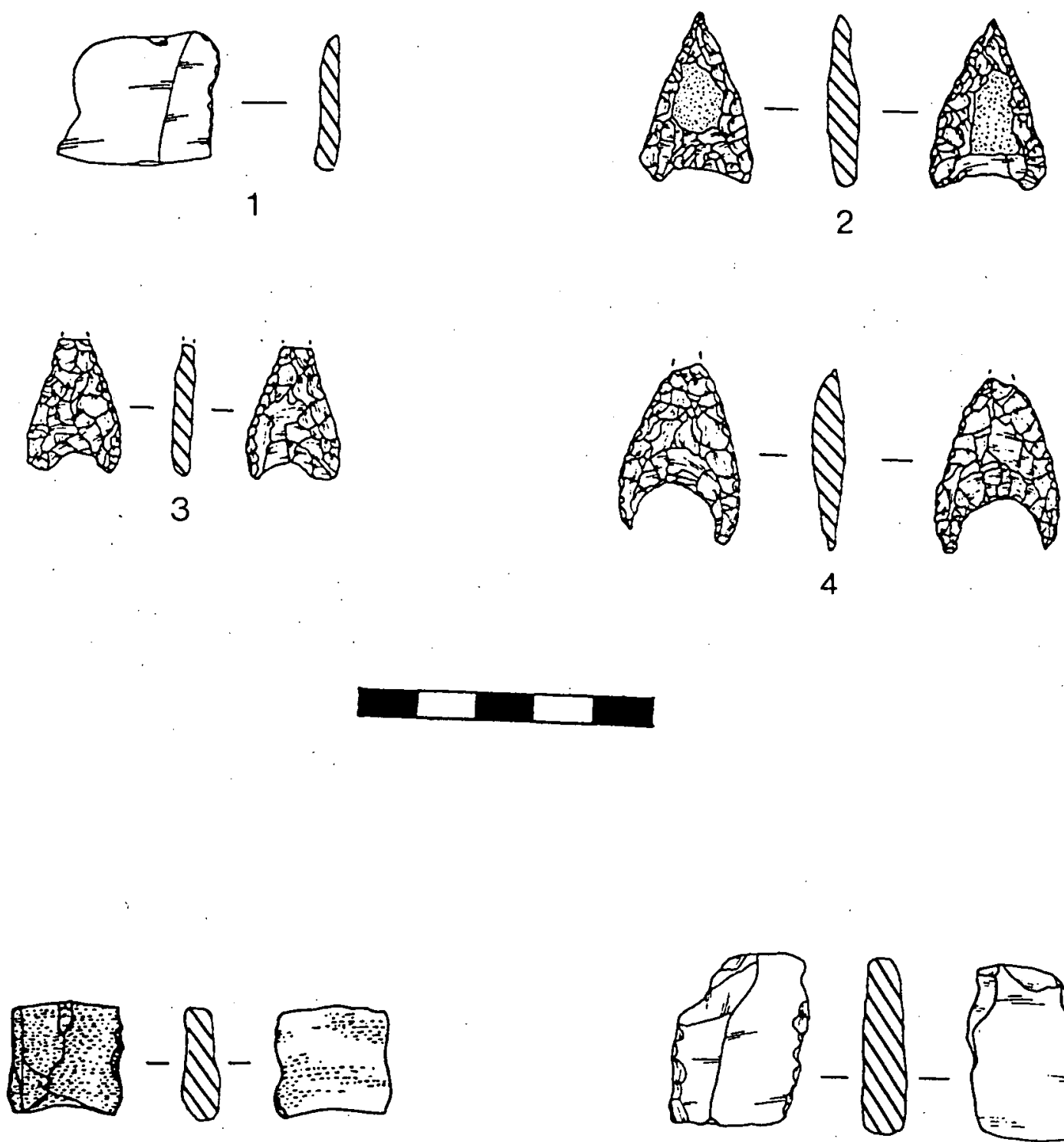

5
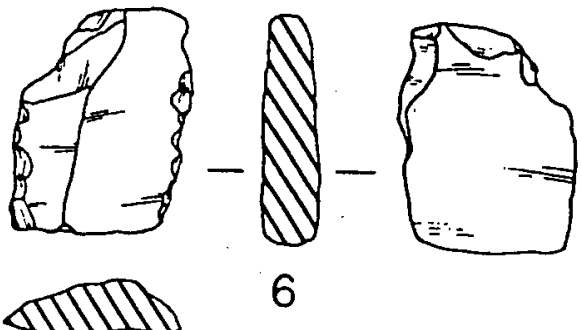

FIGURA 5: 1.- Lámina fracturada con huellas de uso. 2.- Punta foliácea con base simple. 3 y 4.- Puntas foliáceas con alerones. 5.- Elemento de hoz fracturado. 6.- Lámina con retoque abrupto y simple. 